\title{
FATORES ASSOCIADOS AO BAIXO PESO AO NASCER EM CIDADES DA PARAÍBA
}

\author{
FACTORS ATTRIBUTED TO LOW BIRTH WEIGHT IN CITIES OF PARAÍBA
}

\author{
Dixis Figueroa Pedraza ${ }^{a}$ \\ adixisfigueroa@gmail.com \\ Universidade Estadual da Paraíba - Campina Grande (PB), Brasil
}

Data de recebimento do artigo: 31/03/2015

Data de aceite do artigo: 02/10/2015

\section{RESUMO}

Objetivos: Analisar, no período de 1999 a 2007, os fatores associados ao baixo peso ao nascer (BPN) em nascidos vivos de mães residentes nos municípios de Cabedelo e Cacimbas, Paraíba. Métodos: Foram utilizadas as informaçóes do Sistema de Informaçóes sobre Nascidos Vivos (Sinasc). As diferenças no porte populacional e no Índice de Desenvolvimento Humano (IDH) foram as bases principais na escolha dos municípios. Resultados: Os municípios tiveram comportamentos similares em relação aos fatores discriminantes do peso ao nascer. Tanto em Cacimbas quanto em Cabedelo, crianças de mães que fizeram menos de seis consultas de pré-natal e de gestação entre 22 e 36 semanas apresentaram maior chance de BPN. Nos dois municípios, igualmente, a multiparidade representou proteção relacionada com o peso ao nascer. No município de Cabedelo, crianças de mães com idade igual ou superior a 35 anos apresentaram maior probabilidade de BPN. Conclusão: Apesar das limitaçóes das informaçóes disponíveis no Sinasc, seu uso possibilitou identificar fatores comuns do BPN.

Palavras-chave: Recém-nascido de baixo peso; fatores de risco; sistemas de informação.

\section{ABSTRACT}

Objectives: To analyze, in the time period from 1999 to 2007, factors associated with low birth weight (LBW) in liveborn infants of mothers living in the municipalities of Cabedelo and Cacimbas, in the State of Paraíba, Brazil. Method: Information from the Live Birth Information System (SINASC) was used. The differences in the population scale and in the Human Development Index were the main basis for choosing the municipalities. Results: The municipalities had similar behaviors regarding discriminant factors of weight at birth. In Cacimbas, as well as in Cabedelo, children from mothers who underwent less than six prenatal appointments and a pregnancy length from 22 to 36 weeks have shown a greater risk of LBW. In both municipalities, equally, multiparity has represented a protection related to the weight at birth. In the municipality of Cabedelo, children from mothers with age equal or superior to 35 years have shown a greater probability of LBW. Conclusion: In spite of the limited information available on SINASC, its usage has made it possible to identify factors that are common to LBW.

Keywords: Low weight newborns; risk factors; information systems. 


\section{Introdução}

A avaliação de associaçóes do baixo peso ao nascer com indicadores socioeconômicos e de saúde, por exemplo, possibilita uma melhor compreensão espacial e temporal do comportamento das prevalências de baixo peso ao nascer como indicador coletivo de saúde.

A prematuridade (idade gestacional $<37$ semanas) e o crescimento intrauterino restrito, também conhecido como desnutrição fetal (peso ao nascer abaixo do valor limite segundo a idade gestacional), constituem os dois mecanismos principais que condicionam o baixo peso ao nascer $(\text { peso }<2500 \mathrm{~g})^{1}$. Diferente do estimado em estudos anteriores à década de 1990, baseado em 47 estudos de 27 países emergentes, a prematuridade relaciona-se com aproximadamente metade de todos os recém-nascidos com baixo peso ${ }^{2}$. No Brasil, estudos de coorte realizados na cidade de Pelotas confirmam esse comportamento ao apontar aumento de 60\% em 1993 a $68 \%$ em $2004^{3}$. Essa tem sido a principal explicação relacionada às maiores taxas de baixo peso ao nascer nas regiôes mais desenvolvidas no Brasil ${ }^{4}$. Apesar de que o aumento da prematuridade no Brasil vem sendo destacado sem uma identificaçáo clara sobre as causas $^{5}$, indica-se que é reflexo da melhoria na assistência à saúde ${ }^{4}$.

Os resultados anteriores têm representado um avanço importante. Porém, os achados ainda são limitados, uma vez que há carência de estudos populacionais. Além disso, também são poucos os estudos que comparam as taxas de baixo peso ao nascer em municípios de contextos socioeconômicos diferentes da mesma região do país. A abrangência dos dados do Sistema de Informação sobre Nascidos Vivos (Sinasc) possibilitam tais análises ${ }^{6}$. Estimativas a esse respeito são importantes para entender se as diferenças nas taxas de baixo peso ao nascer entre as regióes brasileiras independentes da condição social ${ }^{4}$ apresentam comportamento similar em níveis mais desagregados, como vem sendo questionado 7 .

Este estudo teve como objetivo analisar, no período de 1999 a 2007, fatores associados ao baixo peso ao nascer em nascidos vivos de mães residentes nos municípios de Cabedelo e Cacimbas, Paraíba.

\section{Casuística e métodos}

Trata-se de um estudo a partir de dados secundários para delinear a situaçáo do peso ao nascer nas cidades de Cabedelo e Cacimbas, Paraíba, Nordeste do Brasil, considerando o período de 1999 a 2007. As cidades foram escolhidas segundo suas diferenças pelos critérios de porte populacional e Índice de Desenvolvimento Humano Municipal (IDHM).
A cidade de Cabedelo está situada na zona da mata paraibana, a $18 \mathrm{~km}$ da capital (João Pessoa). O município tem área geográfica de $31 \mathrm{~m}^{2}$, IDHM médio de $0,757,100 \%$ de urbanização, $16,35 \%$ de analfabetismo (população de 15 anos ou mais) e 51.865 habitantes (2009). A cobertura populacional do PACS $\backslash$ PSF municipal é de $100 \%$.

A cidade de Cacimbas está situada na zona do sertão paraibano, a $296 \mathrm{~km}$ da capital (João Pessoa). O município tem área geográfica de $143 \mathrm{~m}^{2}$, IDHM baixo de $0,494,21,48 \%$ de urbanização, $51,08 \%$ de analfabetismo (população de 15 anos ou mais) e 7.059 habitantes (2009). A cobertura populacional do PACS/PSF municipal é de $95,13 \%$.

A população de estudo foi constituída por todos os nascidos vivos de mães residentes nos municípios de Cabedelo e Cacimbas entre 1999 e 2007. Esses anos foram escolhidos considerando que no ano 1998 foi desenhada a última versão da Declaração de Nascidos Vivos (DNV), acompanhada do desenvolvimento de um novo aplicativo informatizado e da elaboraçáo de manuais para sua utilização ${ }^{8}$, bem como a disponibilidade dos dados no momento da elaboraçáo do projeto de pesquisa (até 2007). Foram excluídos os registros sem informação sobre o peso ao nascer, com peso ao nascer $<500 \mathrm{~g}$, sem informação sobre o tipo de gravidez e os de parto gemelar.

Todas as variáveis de interesse da pesquisa foram obtidas a partir do Sinasc referentes aos municípios de Cabedelo e Cacimbas, disponível na página da internet do Departamento de Informática do SUS (Datasus) 9 . Esses dados são provenientes das DNV preenchidas no momento e no local do nascimento, processadas no município e consolidadas no banco de dados da Secretaria Estadual de Saúde, que remete ao Ministério da Saúde para alimentar o Sistema $\mathrm{Nacional}^{10}$. Sendo assim, permitiu-se conhecer o número de nascimentos por município de residência e obter informaçóes sobre as características do recém-nascido (peso ao nascer, sexo, raça/ cor), da gravidez e parto (número de consultas de pré-natal, duração da gestação) e da mãe (idade, situação conjugal, escolaridade, paridade).

O peso ao nascer foi tratado como variável dependente. As análises foram realizadas com a variável categorizada em baixo peso (menor de $2500 \mathrm{~g}$ ) e peso normal (maior ou igual a $2500 \mathrm{~g}$ ). As demais variáveis da DNV objeto de estudo foram consideradas como variáveis independentes preditoras do baixo peso ao nascer.

A força de associação entre as variáveis independentes e o baixo peso ao nascer foi realizada com o cálculo do Odds Ratio - OR com intervalo de confiança 95\%, utilizando regressão logística com abordagem das análises univariada e multivariada. $\mathrm{Na}$ análise univariada as variáveis que obtiveram $\mathrm{p}$-valor $<0,25^{11}$ foram eletivas 
para a multivariada. Na regressão multivariada foi utilizado o método forward e as demais conclusóes foram tomadas ao nível de significância de 5\%. Para a análise comparativa entre as variáveis qualitativas foram aplicados os testes qui-quadrado de Pearson e qui-quadrado de proporçóes, e quando necessário foi utilizado a correção de Yates ou teste exato de Fisher ${ }^{11,12}$. As análises foram realizadas com o programa Rv2.10.0.

\section{Resultados}

Da amostra de 8693 nativivos obtida das declaraçóes de nascidos vivos correspondente ao período de 1999 a 2007 dos municípios de Cabedelo ( $\mathrm{n}=7336)$ e Cacimbas ( $\mathrm{n}=1357)$, foram excluídos 162 registros $(1,86 \%)$, sendo 144 em Cabedelo e 28 em Cacimbas, restando para análise 8531 recém-nascidos de parto único com peso ao nascer $\geq 500 \mathrm{~g}$. Assim, a informação de peso ao nascer foi obtida de 7192 nascidos vivos de máes residentes no município de Cabedelo, $\mathrm{PB}$, e de 1329 nascidos vivos de mães residentes no município de Cacimbas, PB.

A Tabela 1 mostra a força de associação entre as variáveis de estudo e o baixo peso ao nascer. Observa-se que em Cabedelo, à exceção do sexo do recém-nascido, todas as variáveis investigadas mostraram-se estatisticamente associadas ao risco do baixo peso ao nascer. Todas as variáveis analisadas no município de Cabedelo atenderam aos pré-requisitos para análise multivariada.

Tabela 1: Análise dos nascidos vivos segundo fatores associados ao baixo peso ao nascer em crianças de mães residentes nos municípios de Cabedelo e Cacimbas, PB, de 1999 a 2007.

\begin{tabular}{|c|c|c|c|c|c|c|c|c|c|c|}
\hline \multirow[b]{2}{*}{ Variáveis } & \multicolumn{5}{|c|}{ Cabedelo } & \multicolumn{5}{|c|}{ Cacimbas } \\
\hline & $\begin{array}{c}\text { Baixo peso } \\
\text { n (\%) }\end{array}$ & $\begin{array}{c}\text { Normal } \\
\text { n (\%) }\end{array}$ & OR & $\begin{array}{l}\text { IC 95\% } \\
\text { Inf-Sup }\end{array}$ & p-valor & $\begin{array}{c}\text { Baixo peso } \\
\text { n }(\%)\end{array}$ & $\begin{array}{c}\text { Normal } \\
\text { n (\%) }\end{array}$ & OR & $\begin{array}{l}\text { IC 95\% } \\
\text { Inf-Sup }\end{array}$ & p-valor \\
\hline \multicolumn{11}{|c|}{ Características do recém-nascido } \\
\hline Sexo & & & & & & & & & & \\
\hline Masculino & $227(48,3)$ & $3464(51,6)$ & 1,0 & & & $31(44,9)$ & $623(49,6)$ & 1,0 & & \\
\hline Feminino & $243(51,7)$ & $3251(48,4)$ & 1,1 & $1,0-1,4$ & 0,168 & $38(55,1)$ & $632(50,4)$ & 1,2 & $0,7-2,0$ & 0,446 \\
\hline \multicolumn{11}{|l|}{ Raça/cor } \\
\hline Branca & $185(42,0)$ & $3093(50,1)$ & 1,0 & & & $8(36,4)$ & $133(21,3)$ & 1,0 & & \\
\hline Parda & $242(54,9)$ & $2906(47,1)$ & 1,4 & $1,2-1,7$ & $<0,001$ & $13(59,1)$ & $403(64,5)$ & 0,5 & $0,2-1,4$ & 0,176 \\
\hline Outra & $14(3,2)$ & $176(2,9)$ & 1,3 & $0,7-2,3$ & 0,304 & $1(4,6)$ & $89(14,2)$ & 0,2 & $0,1-1,0$ & 0,117 \\
\hline \multicolumn{11}{|c|}{ Características da gravidez e parto } \\
\hline \multicolumn{11}{|l|}{$\begin{array}{c}\text { Consultas de } \\
\text { pré-natal }\end{array}$} \\
\hline$\geq 6$ & $178(39,9)$ & $3770(58,7)$ & 1,0 & & & $9(13,6)$ & $350(28,9)$ & 1,0 & & \\
\hline$<6$ & $268(60,1)$ & $2652(41,3)$ & 2,1 & $1,8-2,6$ & $<0,001$ & $57(86,4)$ & $860(71,1)$ & 2,6 & $1,3-5,6$ & 0,009 \\
\hline \multicolumn{11}{|l|}{$\begin{array}{c}\text { Duraçáo } \\
\text { da gestação } \\
\text { (semanas) }\end{array}$} \\
\hline$\geq 37$ & $193(43,3)$ & $6508(97,8)$ & 1,0 & & & $45(69,2)$ & $1179(95,3)$ & 1,0 & & \\
\hline $22-36$ & $253(56,7)$ & $145(2,2)$ & 58,8 & $4,0-5,7$ & $<0,001$ & $20(30,8)$ & $58(4,7)$ & 9,0 & $4,9-6,1$ & $<0,001$ \\
\hline \multicolumn{11}{|c|}{ Características da mãe } \\
\hline \multicolumn{11}{|l|}{ Idade (anos) } \\
\hline $19-35$ & $334(71,2)$ & $5205(77,6)$ & 1,0 & & & $46(67,7)$ & $865(69,5)$ & 1,0 & & \\
\hline$\leq 18$ & $103(22,0)$ & $1189(17,7)$ & 1,4 & $1,07-1,69$ & 0,011 & $17(25,0)$ & $231(18,6)$ & 1,4 & $0,8-2,4$ & 0,268 \\
\hline$>35$ & $32(6,8)$ & $311(4,6)$ & 1,6 & $1,08-2,31$ & 0,015 & $5(7,3)$ & $149(12,0)$ & 0,6 & $0,2-1,5$ & 0,337 \\
\hline
\end{tabular}




\begin{tabular}{|c|c|c|c|c|c|c|c|c|c|c|}
\hline \multirow[b]{2}{*}{ Variáveis } & \multicolumn{5}{|c|}{ Cabedelo } & \multicolumn{5}{|c|}{ Cacimbas } \\
\hline & $\begin{array}{c}\text { Baixo peso } \\
\text { n }(\%)\end{array}$ & $\begin{array}{c}\text { Normal } \\
\text { n }(\%)\end{array}$ & OR & $\begin{array}{l}\text { IC 95\% } \\
\text { Inf-Sup }\end{array}$ & p-valor & $\begin{array}{c}\text { Baixo peso } \\
\text { n }(\%)\end{array}$ & $\begin{array}{c}\text { Normal } \\
\text { n }(\%)\end{array}$ & OR & $\begin{array}{l}\text { IC 95\% } \\
\text { Inf-Sup }\end{array}$ & p-valor \\
\hline \multicolumn{11}{|l|}{$\begin{array}{l}\text { Situaçáo } \\
\text { conjugal }\end{array}$} \\
\hline $\begin{array}{l}\text { Casada/união } \\
\text { consensual }\end{array}$ & $195(45,4)$ & $3077(50,5)$ & 1,0 & & & $22(33,3)$ & $367(31,2)$ & 1,0 & & \\
\hline Solteira & $231(53,7)$ & $2975(48,8)$ & 1,2 & $1,0-1,5$ & 0,043 & $44(66,7)$ & $800(68,0)$ & 0,9 & $0,6-1,6$ & 0,749 \\
\hline $\begin{array}{l}\text { Outra } \\
\text { (separada/ } \\
\text { viúva) }\end{array}$ & $4(0,9)$ & $41(0,07)$ & 1,5 & $0,5-3,9$ & 0,415 & $0(0,0)$ & $10(0,9)$ & - & - & - \\
\hline \multicolumn{11}{|c|}{ Características da máe } \\
\hline \multicolumn{11}{|l|}{$\begin{array}{l}\text { Escolaridade } \\
\text { (anos) }\end{array}$} \\
\hline 12 ou mais & $51(11,9)$ & $908(15,0)$ & 1,0 & & & $1(1,5)$ & $40(3,5)$ & 1,0 & & \\
\hline 8 a 11 & $125(29,1)$ & $1835(30,4)$ & 1,2 & $0,9-1,7$ & 0,259 & $2(3,0)$ & $97(8,4)$ & 0,8 & $0,1-8,0$ & 0,876 \\
\hline 4 a 7 & $170(39,5)$ & $2278(37,7)$ & 1,3 & $1,0-1,9$ & 0,084 & $17(25,4)$ & $345(29,9)$ & 2,0 & $0,4-6,0$ & 0,515 \\
\hline 1 a 3 & $63(14,7)$ & $818(15,5)$ & 1,4 & $0,9-2,0$ & 0,104 & $27(40,2)$ & $354(30,7)$ & 3,1 & $0,6-5,1$ & 0,280 \\
\hline Nenhuma & $21(4,9)$ & $201(3,3)$ & 1,9 & $1,1-3,1$ & 0,022 & $20(29,9)$ & $317(27,5)$ & 2,5 & $0,5-5,9$ & 0,373 \\
\hline \multicolumn{11}{|l|}{$\begin{array}{c}\text { Paridade } \\
\text { (filhos vivos) }\end{array}$} \\
\hline Nulípara & $225(50,3)$ & $2509(41,3)$ & 1,0 & & & $20(32,3)$ & $286(25,0)$ & 1,0 & & \\
\hline Multípara & $222(49,7)$ & $3571(58,7)$ & 0,7 & $0,6-0,8$ & $<0,001$ & $42(67,7)$ & $858(75,0)$ & 0,7 & $0,4-1,2$ & 0,203 \\
\hline
\end{tabular}

No município de Cacimbas, maior chance de baixo peso ao nascer estatisticamente significante foi encontrado em crianças de mães com menos de 6 consultas de pré-natal durante a gestação em comparação às crianças de mães com 6 ou mais consultas de pré-natal durante a gestação $(p=0,0093)$, e em crianças de mães cuja gestação durou menos de 37 semanas em comparação às crianças de mães cuja gestação durou 37 semanas ou mais $(p=0,0000)$. Essas variáveis, assim como raça/cor e paridade (filhos vivos), foram eleitas para a análise logística multivariada.

A Figura 1 mostra os resultados finais da análise logística multivariada. Observa-se que no município de Cabedelo permaneceram com $\mathrm{p}$-valor $<0,05$, mostrando associação ao baixo peso ao nascer crianças de mães que fizeram menos de seis consultas de pré-natal, com OR (IC 95\%) de 1,51 (1,16-1,97); crianças cuja gestação durou entre 22 e 36 semanas, com uma razão de chances de 55 vezes (IC 95\%: 42,00-72,51); crianças de gestantes com idade igual ou superior a 35 anos, com razão de chances de 1,7 vezes (IC 95\%: 1,01-2,81). As mães multíparas apresentaram OR de 0,74 vezes (IC 95\%: 0,56-0,97), representando uma proteção de menos $26 \%$ quando realizada em comparação com as mães nulíparas.
Figura 1: Análise multivariada da distribuição dos fatores associados ao baixo peso ao nascer em nascidos vivos de mães residentes nos municípios de Cabedelo e Cacimbas, PB, 1999-2007.

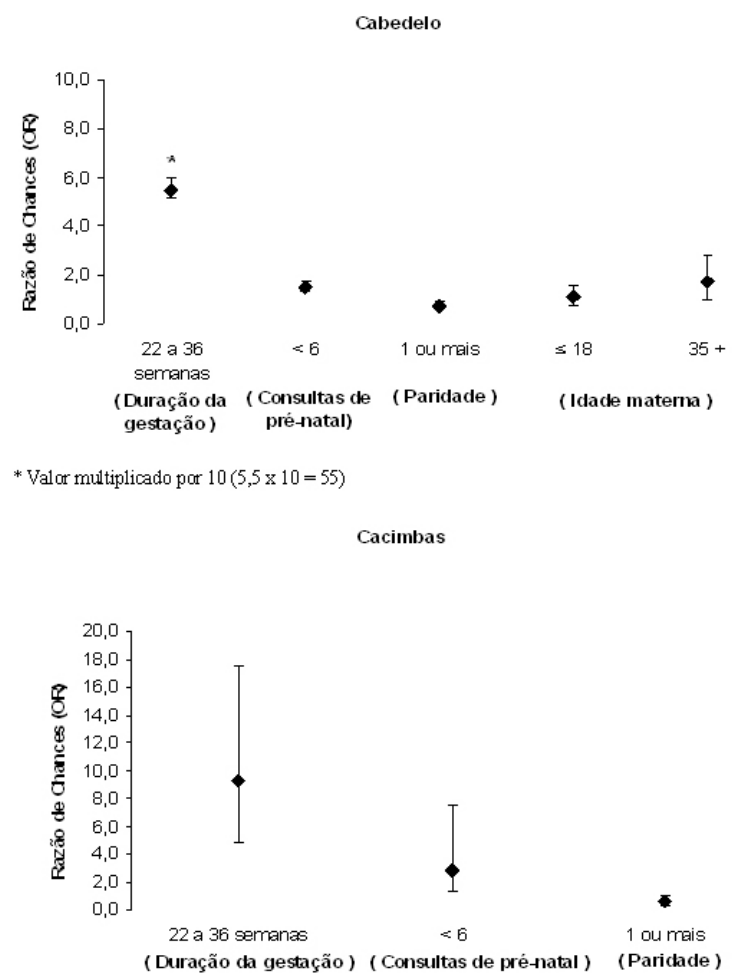


No município de Cacimbas, permaneceram com p-valor $<0,05$, mostrando associação ao baixo peso ao nascer crianças de mães que fizeram menos de seis consultas de pré-natal, com OR (IC 95\%) de 2,84 (1,28$7,53)$ e crianças cuja gestação durou entre 22 e 36 semanas, com uma razão de chances de 9,27 vezes (IC 95\%: 4,80-17,54). As mães multíparas apresentaram OR de 0,54 vezes (IC 95\%: 0,30-1,00), representando uma proteção de menos $46 \%$ quando realizada em comparação com as mães nulíparas.

\section{Discussão}

A influência da duraçáo da gestação no peso ao nascer, bem como a associação entre o baixo número de consultas pré-natal e a ocorrência de baixo peso ao nascer, em Cabedelo e Cacimbas, ratificam os resultados observados em estudos anteriores realizados no Brasi ${ }^{13-17}$. A OMS preconiza que o número de consultas de pré-natal deve ser no mínimo seis, a fim de assegurar às máes a redução dos efeitos adversos de uma assistência pré-natal inadequada ${ }^{18,19}$. O pré-natal é amplamente reconhecido como um dos principais determinantes da evolução gestacional normal. O início do pré-natal, o mais precoce possível, objetiva fortalecer a adesão da mulher ao acompanhamento sistemático ${ }^{20}$, e permitir a detecção e prevenção de complicaçóes obstétricas, por meio de uma intervenção oportuna, sejam elas preventivas ou terapêuticas ${ }^{21}$. Vale a pena ressaltar que a qualidade dessa assistência inclui início precoce, acompanhamento das curvas de peso e altura uterina, avaliação periódica de parâmetros vitais, e entre outros requisitos indispensáveis, que têm como propósito o monitoramento dessas gestantes durante o período gestacional ${ }^{19}$.

Sugere-se que nas regiōes mais desenvolvidas do Brasil as maiores taxas de baixo peso ao nascer, condicionadas por maiores taxas de nascimento pré-termo, ocorram devido às melhores condiçóes para detectar problemas durante o pré-natal, que implica em maior intervenção médica e sobrevida ${ }^{4}$. Fatores associados à prematuridade, como o estado nutricional materno e condiçóes da gravidez (estilo de vida, intercorrências, assistência pré-natal), têm sido estabelecidos em estudos pontuais, sem conseguir explicar o aumento dos casos no Brasil'5. Nesse contexto, parece ser que o parto cesariano constitui um evento importante $e^{1,4,5,22}$. Enquanto alguns estudos mostram que no nascimento pré-termo as intervenções médicas, como as cesarianas, têm uma importante contribuição, outros indicam que a prematuridade aumenta tanto nos nascimentos por cesariana quanto por parto vaginal ${ }^{22}$. Entretanto, análises nacionais apontam o aumento das taxas de baixo peso ao nascer com o aumento das taxas de cesariana ${ }^{4}$, cuja frequência é maior entre mulheres de grupos socioeconômicos mais privilegiados ${ }^{22}$. Assim, dada a atual conjuntura que indica o importante papel da prematuridade no baixo peso ao nascer e na mortalidade infantil, o conhecimento sobre as causas do aumento de partos prematuros no Brasil torna-se relevante.

Neste estudo, as mulheres multíparas com paridade entre 1-3 filhos representaram 94,2\% das mulheres multíparas (2085, 1101 e 387 casos de mães com 1, 2 e 3 nascidos vivos, respectivamente). A paridade entre 1-3 filhos representa a melhor condiçáo relacionada ao peso ao nascer da criança (categoria de referência) ${ }^{15}$, o que adere à proteção que representou as mães com um ou mais filhos vivos nos municípios de Cabedelo e Cacimbas. Estudos indicam maior prevalência de baixo peso ao nascer entre as mulheres nulíparas e as grandes multíparas ${ }^{15,23}$. Sugere-se que mulheres nulíparas apresentam maior probabilidade de gestação pré-termo, enquanto mulheres multíparas apresentam maior probabilidade ao desencadeamento de crianças pequenas para idade gestacional ${ }^{23,24}$. Entretanto, as razóes explicativas não são suficientes.

Estudos desenvolvidos em diversos municípios brasileiros ${ }^{13-15,17,24,25}$ evidenciaram a associação observada no município de Cabedelo entre o baixo peso ao nascer e a idade materna de 35 anos ou mais. Essa associaçáo parece estar relacionada à maior incidência de complicações clínicas e obstétricas nessa faixa etária. Essas gestantes são substancialmente mais propensas a ter intercorrências na gravidez como diabetes, hipertensão, gestação múltipla, parto prematuro, rotura prematura das membranas e hemorragia anteparto. Estas patologias modificam o intercurso da gestação e aumentam as ocorrências de partos prematuros ${ }^{17,26,27}$.

Os resultados deste estudo não devem ser vistos como de interesse somente local, pois a avaliaçáo do comportamento das proporçôes de baixo peso ao nascer em nível local pode levar a uma melhor compreensão do mesmo como indicador coletivo de saúde. As variaçóes nas proporçóes de baixo peso ao nascer podem ocorrer devido aos efeitos da localidade de moradia da mãe do nascido vivo ${ }^{1}$ (semelhanças por compartilhamento do mesmo ambiente ou ambientes similares podem levar, também, a uma maior semelhança no desfecho).

Conclui-se que, apesar das limitaçóes das informaçôes disponíveis no Sinasc, seu uso possibilitou identificar a inadequação do pré-natal e a prematuridade como fatores comuns associados ao baixo peso ao nascer em municípios de contextos populacionais e socioeconômicos diferentes. A influência da prematuridade no baixo peso ao nascer exige um melhor entendimento. Como recém-nascidos prematuros são crianças que demandam cuidados especiais, esses resultados devem ter importantes reflexos no planejamento em saúde. 


\section{Referências}

1. Moraes AB, Zanini RR, Giugliani ERJ, Riboldi J. Tendência da proporção de baixo peso ao nascer, no período de 19942004, por microrregião do Rio Grande do Sul, Brasil: uma análise multinível. Cad Saúde Pública. 2011;27(2):229-40.

2. Barros FC, Barros AJD, Villar J, Matijasevich A, Domingues MR, Victora CG. How many low birthweight babies in low and middle income countries are preterm? Rev Saúde Pública. 2011;45(3):607-16.

3. Barros FC, Victora CG, Matijasevich A, Santos IS, Horta BL, Silveira MF, et al. Preterm births, low birth weight, and intrauterine growth restriction in three birth cohorts in Southern Brazil: 1982, 1993 and 2004. Cad Saúde Pública. 2008;24(Suppl 3):S390-8.

4. Silva AAM, Silva LM, Barbieri MA, Bettiol H, Carvalho LM, Ribeiro VS, et al. The epidemiologic paradox of low birth weight in Brazil. Rev Saúde Pública. 2010;44(5):767-75.

5. Silveira MF, Santos IS, Barros AJD, Matijasevich A, Barros FC, Victora CG. Aumento da prematuridade no Brasil: revisão de estudos de base populacional. Rev Saúde Pública. 2008;42:957-64.

6. Andrade CLT, Szwarcwald CL, Castilho EA. Baixo peso ao nascer no Brasil de acordo com as informaçóes sobre nascidos vivos do Ministério da Saúde, 2005. Cad Saúde Pública. 2008;24(11):2564-72.

7. Robin L, Young RL, Weinberg J, Vieira V, Aschengrau A, Webster TF. A multilevel non-hierarchical study of birth weight and socioeconomic status. Int J Health Geogr. 2010;9(36):1-12.

8. Fundação Nacional de Saúde. Manual de procedimentos do Sistema de Informaçóes sobre Nascidos Vivos. Brasília: Funasa; 2001.

9. Costa JMBS, Frias PG. Avaliação da completitude das variáveis da Declaração de Nascido Vivo de residentes em Pernambuco, Brasil, 1996 a 2005. Cad Saúde Pública. 2009;25(3):613-24.

10. Vidal SA, Arruda BKG, Vanderlei LC, Frias PG. Avaliação da série histórica dos nascidos vivos em unidade terciária de Pernambuco - 1991 a 2000. Rev Assoc Med Bras. 2005;51(1):17-22.

11. Jerrold HZ. Biostatistical analysis. $3^{\mathrm{a}}$ ed. New Jersey: Prentice Hall; 1996.

12. Hosmer DW, Lemeshow S. Applied logistic regression. $2^{\text {a }}$ ed. New York: John Wiley and Sons; 2000.

13. Carniel EF, Zanoli ML, Antônio MARGM, Morcillo AM. Determinantes do baixo peso ao nascer a partir das declaraçóes de nascidos vivos. Rev Bras Epidemiol. 2008;11(11):169-79.

14. Guimarães EAA, Velásquez-Melendez G. Determinantes do baixo peso ao nascer a partir do Sistema de Informação sobre nascidos vivos em Itaúna, Minas Gerais. Rev Bras Saude Matern Infant. 2002;2(3):283-90.

15. Uchimura TT, Pelissari DM, Uchimura NS. Baixo peso ao nascer e fatores associados. Rev Gaucha Enferm. 2008;29(1):33-8.

16. Barbas DS, Costa AJL, Luiz RR, Kale PL. Determinantes do peso insuficiente e do baixo peso ao nascer na cidade do Rio de Janeiro, Brasil, 2001. Epidemiol Serv Saúde. 2009;18(2):161-70.

17. Giglio MRP, Lamounier JA, Neto OLM, César CM. Baixo peso ao nascer em coorte de recém-nascidos em Goiânia-Brasil no ano de 2000. Rev Bras Ginecol Obstet. 2005;27(3):130-6.

18. Oliveira NC, Moura ERF, Silva CF. Perfil dos nascidos vivos de Maranguape - CE: a partir da série de 2000 a 2003. Rev RENE. 2007;8(1):52-9.

19. Costa MCO, Santos CAT, Sobrinho CL, Freitas JO, Ferreira KASL. Indicadores materno-infantis na adolescência e juventude: sócio-demográfico, pré-natal, parto e nascidos-vivos. J Pediatr. 2001;77(3):235-42.

20. Grangeiro GR, Diógenes MAR, Moura ERF. Atenção pré-natal no município de Quixadá-CE segundo indicadores de processo do Sisprenatal. Rev Esc Enferm USP. 2008;42(1):105-11.

21. Spindola T, Penna LHG, Progianti JM. Perfil epidemiológico de mulheres atendidas na consulta do pré-natal de um hospital universitário. Rev Esc Enferm USP. 2006;40(3):381-8.

22. Victora CG, Aquino EML, Leal MC, Monteiro CA, Barros FC, Szwarcwald CL. Saúde de mães e crianças no Brasil: progressos e desafios. The Lancet [periódico na Internet]. 2011 Mai [acesso em 2012 Ago 26];32-46. Disponível em: http://download.thelancet.com/flatcontentassets/pdfs/brazil/brazilpor2.pdf.

23. Minuci EG, Almeida MF. Diferenciais intra-urbanos de peso ao nascer no município de São Paulo. Rev Saúde Pública. 2009;43(2):256-66.

24. Costa EC, Gotlieb SLD. Estudo epidemiológico do peso ao nascer a partir da Declaraçáo de Nascidos Vivos. Rev Saúde Pública. 1998;32(4):328-34.

25. Nascimento LFC, Gotlieb SLD. Fatores de risco para o baixo peso ao nascer, com base em informaçóes da Declaração de Nascido Vivo em Guaratinguetá, SP, no ano de 1998. Inf Epidemiol SUS. 2001;10(3):113-20.

26. Ziadeh SM. Maternal and perinatal outcome in nulliparous women aged 35 and older. Gynecol Obstet Invest. 2002;54(1):6-10.

27. Senesi LG, Tristão EG, Andrade RP, Krajden ML, Junior FCO, Nascimento DJ. Morbidade e mortalidade neonatais relacionadas à idade materna igual ou superior a 35 anos, segundo a paridade. RBGO. 2004;26(6):477-82.

\section{Como citar este artigo:}

Pedraza DF. Fatores associados ao baixo peso ao nascer em cidades da Paraíba. Rev. Aten. Saúde. 2016;14(47): 43-48. 